\title{
Quantification of table olives' acid, bitter and salty tastes using potentiometric electronic tongue fingerprints
}

\author{
Ítala M.G. Marx ${ }^{\mathrm{a}, \mathrm{b}}$, Nuno Rodrigues ${ }^{\mathrm{c}, \mathrm{d}}$, Luís G. Dias ${ }^{\mathrm{a}, \mathrm{e}}$, Ana C.A. Veloso ${ }^{\mathrm{f}, \mathrm{g}}$, \\ José A. Pereira ${ }^{\mathrm{h}}$, Deisy A. Drunkler ${ }^{\mathrm{b}}$, António M. Peres ${ }^{\mathrm{i}, *}$ \\ a School of Agriculture, Polytechnic Institute of Bragança, Campus Santa Apolónia, 5300-253 Bragança, Portugal \\ b Universidade Tecnológica Federal do Paraná-UTFPR, Câmpus Medianeira, Avenida Brasil, 4232, Parque Independência, 85884-000, Medianeira, Paraná, \\ Brazil \\ ${ }^{c}$ REQUIMTE-LAQV/CIMO, School of Agriculture, Polytechnic Institute of Bragança, Campus Santa Apolónia, $5300-253$ Bragança, Portugal \\ d Universidad de Léon, Departamento de Ingeniería Agrária, Av. Portugal, $n^{\circ}$ 41, 24071 Léon, Spain \\ e CQ-VR, Centro de Química - Vila Real, University of Trás-os-Montes e Alto Douro, Apartado 1013, 5001-801 Vila Real, Portugal \\ ${ }^{\mathrm{f}}$ Instituto Politécnico de Coimbra, ISEC, DEQB, Rua Pedro Nunes, Quinta da Nora, 3030-199 Coimbra, Portugal \\ ${ }^{g}$ CEB - Centre of Biological Engineering, University of Minho, Campus de Gualtar, 4710-057 Braga, Portugal \\ ${ }^{\text {h }}$ REQUIMTE-LAQV, School of Agriculture, Polytechnic Institute of Bragança, Campus Santa Apolónia, 5300-253 Bragança, Portugal \\ ${ }^{\mathrm{i}}$ Laboratory of Separation and Reaction Engineering - Laboratory of Catalysis and Materials (LSRE-LCM), Escola Superior Agrária, Instituto Politécnico de \\ Bragança, Campus Santa Apolónia, 5300-253 Bragança, Portugal
}

\section{A R T I C L E I N F O}

\section{Article history:}

Received 22 October 2016

Received in revised form

18 January 2017

Accepted 19 January 2017

Available online 21 January 2017

\section{Keywords:}

Table olives

Basic taste intensity

Electronic tongue

Linear discriminant analysis

Multiple linear regression

\begin{abstract}
A B S T R A C T
The intensities of the gustatory attributes of table olives is one of the sensory set of parameters evaluated by trained sensory panels accordingly to the recommendations of the International Olive Council. However this is an expensive and time-consuming process that only allows the evaluation of a limited number of samples per day. So, an electronic tongue coupled with multivariate statistical tools, is proposed for assessing the median intensities of acid, bitter and salty tastes perceived in table olives. The results showed that the device, coupled with linear discriminant analysis, could be used as a taste sensor, allowing classifying aqueous standard solutions according to the three basic tastes (repeated $K$-fold cross-validation: $98 \% \pm 3 \%$ of correct classifications) based on the electrochemical signals of 5 sensors. It was demonstrated that the taste sensor with multiple linear regression models, enabled quantifying the median intensities of the three basic tastes (repeated $K$-fold cross-validation: $R^{2} \geq 0.96 \pm 0.04$ ) perceived in table olives by a trained sensory panel, based on the potentiometric fingerprints (21-25 signal profiles) of aqueous olive pastes and brines. The overall satisfactory results showed the electronic tongue potential to assess the intensities of gustatory attributes of table olives, formerly only achievable by sensory panels.
\end{abstract}

(c) 2017 Elsevier Ltd. All rights reserved.

\section{Introduction}

Table olives are produced from healthy fruits of the species Olea europaea L., after complete or partial fermentation. The fermentation may be monitored by chemical, physico-chemical and microbiological procedures as well as by the sensory evaluation performed by trained sensory panels (IOC, 2011). Within the sensory analysis, gustatory sensations like salty, bitter and acid must be evaluated, involving distinct areas of the human tongue (Lanza \&

\footnotetext{
* Corresponding author.

E-mail address: peres@ipb.pt (A.M. Peres).
}

Amoruso, 2016). The sensory evaluation of the gustatory attributes is made in each end-product (table olives and brines) by official or trained sensory panels (minimum of 8 panelists), using an intensity-scale ranging from 1.0 (absence of sensation) to 11.0 (extremely intense sensation), and is limited in time and number of samples that can be daily evaluated (IOC, 2004). Despite the cost and time required for training and sensory evaluation, the use of sensory panels, trained according to the International Olive Council directives (IOC, 2011), is still the most common method for assessing the sensory characteristics of table olives, which are then used by researchers for deeper understanding changes that occur during table olives' production or storage (e.g., Blana, Polymeneas, Tassou, \& Panagou, 2016; Cano-Lamadrid et al., 2016; Campus 
et al., 2015; De Angelis et al., 2015; Lanza \& Amoruso, 2016; LópezLópez, Bautista-Gallego, Moreno-Baquero, \& Garrido-Fernández, 2016; Martorana et al., 2016; Randazzo et al., 2014; RodríguezGoméz et al., 2014). However, aiming that, in a near future the sensory evaluation of table olives goes from a simple recommendation of the IOC to a legal requirement, and knowing that only 3 samples per session ( 3 session by day) may be evaluated by a trained sensory panel, it is required to develop and validate novel, fast and low-cost analytical techniques that may allow increasing the total number of samples assessed per day and, therefore, to fulfill this future practical need. Electrochemical based-sensors, namely electronic tongues (E-tongue), have emerged in the last years as powerful taste sensor devices that allow successful overall qualitative and/or quantitative sensory analysis of basic gustatory attributes (namely, acid, bitter, salty and sweet) and/or sensory defects, either in standard solutions of chemical compounds that mimic those gustatory sensations or in food/beverages samples, including beer, coffee, fruit juice, green tea, meat, milk, olive oil, rice, soy sauce, soup and wines (Apetrei et al., 2010; Apetrei, Ghasemi-Vernamkhasti, \& Apetrei, 2016; Iiyama, Yahiro, \& Toko, 2000; Borràs et al., 2016; Dias et al., 2009; Dias, Sequeira, Veloso, Sousa, \& Peres, 2014; Dias, Rodrigues, Veloso, Pereira, \& Peres, 2016; Mizota et al., 2009; Rodríguez-Méndez, Apetrei, \& de Saja, 2010; Toko, 2013; Veloso, Dias, Rodrigues, Pereira, \& Peres, 2016). Concerning the application of taste sensor devices for table olives sensory evaluation, and to the authors' best knowledge, few works have been reported in the literature. Panagou, Sahgal, Magan and Nychas (2008) investigated the potential application of an electronic nose (E-nose) for quality discrimination of fermented table olives, according to 3 classes (acceptable, unacceptable and marginal) established based on the sensory evaluation performed by a trained sensory panel. Recently, Marx et al. (2017) used an E-tongue to classify table olives according to trade sensory quality classes, being the potentiometric device also able to distinguish negative sensory attributes (e.g., butyric, putrid, zapateria, musty and winevinegary sensations) perceived in table olives by a trained sensory panel. In this work, for the first time, the capability of an E-tongue, comprising cross-sensitivity lipid polymeric membranes, to quantify the sensory intensity of acid, bitter and salty sensations in table olives (i.e., descriptive gustatory attributes that must be evaluated according to the IOC recommendation) was investigated based on the potentiometric signals recorded during the analysis of aqueous olive pastes and respective brines, mimicking the procedure followed by the trained panelists. Indeed, in the past, taste sensors based on artificial lipids showed satisfactory global selectivity towards basic tastes and high correlation to human sensory scores, allowing to detect taste-substance interactions (synergistic and suppression effects) and to minimize physical and psychological conditions and individual preference of panelists (Kobayashi et al., 2010; Toko, 2013).

\section{Materials and methods}

\subsection{Table olives samples}

Forty-four commercial table olives samples, from 18 different brands, were purchased in local supermarkets in Bragança (Portugal) and Zamora (Spain). The olive cultivars and the technological process applied for table olives production have been previously reported (Marx et al., 2017).

\subsection{Table olives sensory analysis: sensory panel and sample preparation}

The sensory assessment of table olives was performed by a trained sensory panel from Escola Superior Agrária-Instituto Politécnico de Bragança, which evaluated and graded each sample (olive and brine solution) according to a pre-defined intensity scale that varied from 1 (sensory sensation not perceived) to 11 (sensation intensity extremely perceived). Previously, olfactory and/or gustatory negative attributes of those table olives were evaluated enabling classifying these table olives based on the median intensity of the defect predominantly perceived (DPP) as extra without defect, extra, $1^{\text {st }}$ choice, $2^{\text {nd }}$ choice and olives that cannot be commercialized as table olives (Marx et al., 2017). In this work, descriptive gustatory attributes were evaluated by the sensory panel following the IOC recommendations (IOC, 2011): acid sensation (basic taste produced by dilute aqueous solutions of substances like tartaric and citric acids), bitter sensation (basic taste produced by dilute aqueous solutions of substances like quinine or caffeine) and salty sensation (basic taste produced by dilute aqueous solutions of substances like sodium chloride).

Table 1

E-tongue sensors details (identification code; pairs of plasticizer additive compounds, used in the preparation of each lipid-polymeric membrane) (Marx et al., 2017).

\begin{tabular}{|c|c|c|c|}
\hline \multicolumn{2}{|c|}{ Sensor code } & \multirow[t]{2}{*}{ Plasticizer ( 65\%) } & \multirow[t]{2}{*}{ Adittive ( $3 \%)$} \\
\hline $1^{\text {st }}$ array & $\overline{2^{\text {nd }}}$ array & & \\
\hline $\mathrm{S} 1: 1$ & $\mathrm{~S} 2: 1$ & 2-Nitrophenyl-octyl ether & Octadecylamine \\
\hline $\mathrm{S} 1: 2$ & $\mathrm{~S} 2: 2$ & & Oleyl alcohol \\
\hline $\mathrm{S} 1: 3$ & $\mathrm{~S} 2: 3$ & & Methyltrioctylammonium chloride \\
\hline $\mathrm{S} 1: 4$ & $\mathrm{~S} 2: 4$ & & Oleic acid \\
\hline $\mathrm{S} 1: 5$ & $\mathrm{~S} 2: 5$ & Tris(2-ethyl-hexyl) phosphate & Octadecylamine \\
\hline S1:6 & S2:6 & & Oleyl alcohol \\
\hline $\mathrm{S} 1: 7$ & S2:7 & & Methyltrioctylammonium chloride \\
\hline $\mathrm{S} 1: 8$ & $\mathrm{~S} 2: 8$ & & Oleic acid \\
\hline S1:9 & S2:9 & Bis(1-butylpentyl) adipate) & Octadecylamine \\
\hline S1:10 & $\mathrm{S} 2: 10$ & & Oleyl alcohol \\
\hline $\mathrm{S} 1: 11$ & S2:11 & & Methyltrioctylammonium chloride \\
\hline $\mathrm{S} 1: 12$ & $\mathrm{~S} 2: 12$ & & Oleic acid \\
\hline $\mathrm{S} 1: 13$ & S2:13 & Dibutyl sebacate & Octadecylamine \\
\hline S1:14 & S2:14 & & Oleyl alcohol \\
\hline S1:15 & S2:15 & & Methyltrioctylammonium chloride \\
\hline S1:16 & S2:16 & & Oleic acid \\
\hline S1:17 & S2:17 & Bis(2-ethylhexyl)phthalate & Octadecylamine \\
\hline $\mathrm{S} 1: 18$ & $\mathrm{~S} 2: 18$ & & Oleyl alcohol \\
\hline $\mathrm{S} 1: 19$ & S2:19 & & Methyltrioctylammonium chloride \\
\hline $\mathrm{S} 1: 20$ & $\mathrm{~S} 2: 20$ & & Oleic acid \\
\hline
\end{tabular}


Table 2

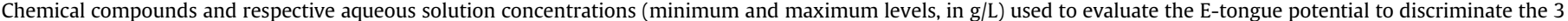

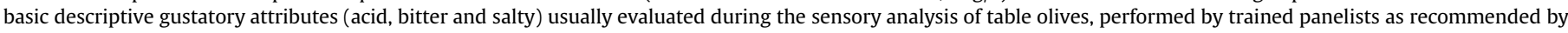

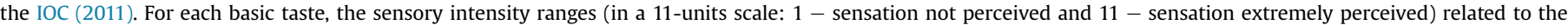
minimum and maximum concentrations are also given.

\begin{tabular}{|c|c|c|c|}
\hline Descriptive gustatory attribute & Standard compound (chemical formula) & $\begin{array}{l}\text { Concentration range } \\
(\mathrm{g} / \mathrm{L})\end{array}$ & Sensory intensity \\
\hline Acid & $\begin{array}{l}\text { Citric acid }\left(\mathrm{C}_{6} \mathrm{H}_{8} \mathrm{O}_{7}\right) \\
\text { Tartaric acid }\left(\mathrm{C}_{4} \mathrm{H}_{6} \mathrm{O}_{6}\right)\end{array}$ & $\begin{array}{l}0.125 \text { to } 2 \\
0.01 \text { to } 0.5\end{array}$ & 2.5 to 7.0 \\
\hline Bitter & $\begin{array}{l}\text { Caffeine }\left(\mathrm{C}_{8} \mathrm{H}_{10} \mathrm{~N}_{4} \mathrm{O}_{2}\right) \\
\text { Anhydrous Quinine }\left(\mathrm{C}_{20} \mathrm{H}_{24} \mathrm{~N}_{2} \mathrm{O}_{2}\right)\end{array}$ & $\begin{array}{l}0.15 \text { and } 3 \\
0.01 \text { to } 0.1\end{array}$ & 4.5 to 9.5 \\
\hline Salty & $\begin{array}{l}\text { Sodium chloride }(\mathrm{NaCl}) \\
\text { Potassium chloride }(\mathrm{KCl})\end{array}$ & $\begin{array}{l}0.5 \text { and } 15 \\
2 \text { to } 25\end{array}$ & 3.5 to 10.0 \\
\hline
\end{tabular}

\subsection{E-tongue device}

The E-tongue multi-sensor was previously described (Marx et al., 2017). Briefly, it comprised two print-screen potentiometric arrays $(9.5 \mathrm{~cm} \times 2.5 \mathrm{~cm})$ containing each 20 sensors $(3.6 \mathrm{~mm}$ of diameter, $0.3 \mathrm{~mm}$ of thickness). The E-tongue sensors included lipid additives $(\approx 3 \%)$, plasticizer compounds $(\approx 65 \%)$ and high molecular weight polyvinyl chloride (PVC, $\approx 32 \%$ ), chosen due to their signal stability over time and repeatability towards the basic standard taste compounds (sweet, acid, bitter, salty and umami) (Dias et al., 2009). Each sensor was coded with a letter S (for sensor) followed by a code for the sensor array (1: or 2:) and the number of the membrane (1-20), corresponding to different combinations of plasticizer and additive compounds, as shown in Table 1.

\subsection{E-tongue assays}

\subsubsection{Standard solutions mimicking descriptive gustatory attributes of table olives}

Aqueous standard solutions (Table 2) were used to mimic the 3 descriptive gustatory sensations usually perceived in table olives and respective brine solutions: acid basic taste was mimicked using citric acid (Fisher Scientific, p.a.) and ( $\mathrm{L}+$ )-tartaric acid (Panreac, p.a.), bitter sensation was described using quinine (Fluka, $\geq 98 \%$ ) and caffeine (Panreac, p.a.), and salty attribute was simulated using sodium chloride and potassium chloride (Panreac, p.a.). Nine concentrations of each standard aqueous solution were used, including the recommended concentration ranges used in sensory panel training (Meilgaard, Civille, \& Carr, 2007). The standard solutions were prepared using commercial mineral water $(\mathrm{pH}=5.8 \pm 0.2$; total minerals $=48 \pm 7 \mathrm{mg} / \mathrm{L}$ ) to simulate the solutions assessed by the sensory panel (for which deionised or distilled water cannot be used). The potentiometric response of each E-tongue sensor towards the concentration of the each aqueous standard solution was evaluated by calculating the sensor sensitivity (in $\mathrm{mV} /$ decade), through the plot of the signal versus the decimal logarithm of the solution concentration. The repeatability of the signals of the Etongue was also checked repeating the electrochemical measurement of a selected aqueous standard solutions (i.e., that with the median concentration of the ranges indicated in Table 2) three times in a day ( $5 \mathrm{~h}$ interval).

Finally, each electrochemical assay required $20 \mathrm{~mL}$ of each aqueous standard solution, being all solutions analyzed at controlled temperature $\left(\sim 20^{\circ} \mathrm{C}\right)$ under agitation using a magnetic stirrer (Velp Scientifica). The E-tongue was immersed directly in each solution and after 5 min stabilization period the potentiometric signal profiles the 40 E-tongue lipid membranes were acquired.

\subsubsection{Table olives: aqueous olive pastes and brine solutions}

The 44 table olive samples were also electrochemically analyzed using the E-tongue. The analysis of olives required a preliminary pre-treatment step to obtain an aqueous olive paste. First, olive stones were removed and then the pulp was crushed using shredder knives Moulinex ${ }^{\circledR}$ equipment. The process turned out into a fine-grained paste, which was diluted using mineral water ( $9 \mathrm{~g}$ of table olive paste diluted in $6 \mathrm{~mL}$ mineral water, the same water used for the sensory panel training). For the brine solutions, $20 \mathrm{~mL}$ of each sample were directed analyzed (Marx et al., 2017).

\subsection{Statistical analysis}

Statistical differences between the median intensity of the descriptive gustatory attributes (acid, bitter and salty) between the table olives' quality trade categories previously established by Marx et al. (2017) were investigated using one-way ANOVA followed, when appropriate, by the Tukey's post-hoc multi-comparison test.

Linear discriminant analysis (LDA) and multiple linear

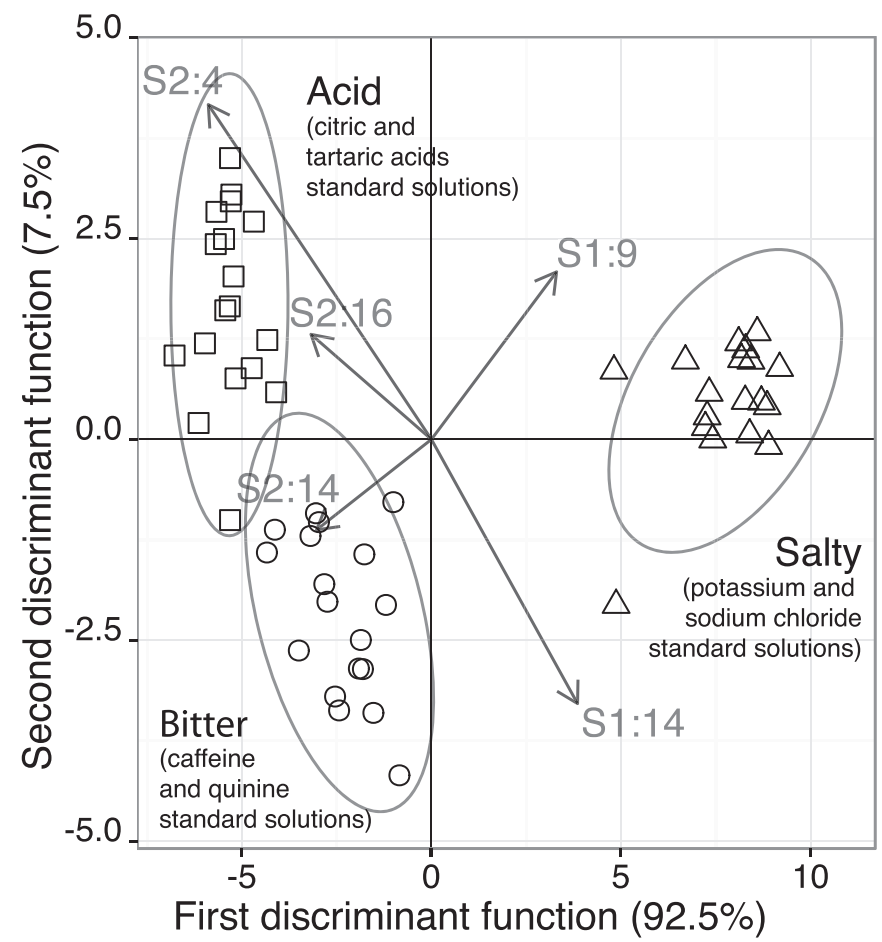

Fig. 1. Discrimination of aqueous standard chemical solutions used to mimic descriptive gustatory attributes evaluated during table olives sensory analysis (IOC, 2011) using an E-tongue-LDA-SA model based on the potentiometric signals of 5 sensors of the E-tongue (S1:9, S1:14, S2:4, S2:14, e S2:16). Symbol for acid basic taste (citric acid: $0.125-2 \mathrm{~g} / \mathrm{L}$; tartaric acid: $0.01-0.5 \mathrm{~g} / \mathrm{L}$ ); symbol $\bigcirc$ for bitter basic taste (for caffeine: $0.15-3 \mathrm{~g} / \mathrm{L}$; quinine: $0.01-0.1 \mathrm{~g} / \mathrm{L}$ ); and, symbol $\Delta$ for salty basic taste (potassium chloride: $2-25 \mathrm{~g} / \mathrm{L}$; sodium chloride: $0.5-15 \mathrm{~g} / \mathrm{L}$ ). 
Table 3

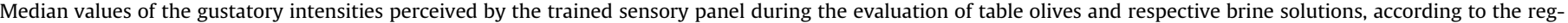
ulations of the IOC (2011). The range of intensities perceived by 8 panellists (minimum and maximum intensity levels) is given in brackets.

\begin{tabular}{|c|c|c|c|c|}
\hline Sample number & Acid basic taste & Bitter basic taste & Salty basic taste & Trade category classification ${ }^{a}$ \\
\hline 1 & $5(5-5.5)$ & $5(5-5.5)$ & $6(5-6.5)$ & Extra \\
\hline 2 & $6(5.5-6)$ & $5.5(5.5-6)$ & $6(5-6.5)$ & $1^{\text {st }}$ choice \\
\hline 3 & $5.5(5-6)$ & $5(5-6.5)$ & $6(5-6.5)$ & Extra \\
\hline 4 & $5.5(5-5.5)$ & $5.5(5-5.5)$ & $6(5.5-6.5)$ & Extra \\
\hline 5 & $5(5-5.5)$ & $6.25(6-6.5)$ & $6(5-6.5)$ & $\mathrm{NC}$ \\
\hline 6 & $5.5(5.5-6)$ & $5.5(5.5-6)$ & $6(5-6.5)$ & $1^{\text {st }}$ choice \\
\hline 7 & $5.5(5-5.5)$ & $5(5-5.5)$ & $6(5-6.5)$ & $2^{\text {nd }}$ choice \\
\hline 8 & $5.5(5-5.5)$ & $5(5-5.5)$ & $5.5(4.5-6.5)$ & $2^{\text {nd }}$ choice \\
\hline 9 & $5(5-5.5)$ & $5.5(5-5.5)$ & $6(5-6.5)$ & $2^{\text {nd }}$ choice \\
\hline 10 & $6(5-6)$ & $5.5(5-6)$ & $5.5(5-6.5)$ & $1^{\text {st }}$ choice \\
\hline 11 & $6(6-6.5)$ & $5(5-5.5)$ & $6(5-6.5)$ & Extra \\
\hline 12 & $5.75(5-6)$ & $5.25(5-6)$ & $6(5-6.5)$ & $1^{\text {st }}$ choice \\
\hline 13 & $5.5(5-5.5)$ & $4(3.5-4)$ & $5(5-5.5)$ & Extra \\
\hline 14 & $6(5.5-6)$ & $5(5-5.5)$ & $6(5-6.5)$ & $1^{\text {st }}$ choice \\
\hline 15 & $4.5(4-4.5)$ & $4.25(4-4.5)$ & $5(5-6.5)$ & $\mathrm{NC}$ \\
\hline 16 & $5.5(5-5.5)$ & $5(5-5.5)$ & $6(5-6.5)$ & $2^{\text {nd }}$ choice \\
\hline 17 & $5(4-5)$ & $4.5(4-4.5)$ & $6.5(6-6.5)$ & $1^{\text {st }}$ choice \\
\hline 18 & $5.5(5-5.5)$ & $5(5-5)$ & $6.25(5-6.5)$ & $1^{\text {st }}$ choice \\
\hline 19 & $5(5-5.5)$ & $5(5-5.5)$ & $6.25(6-6.5)$ & $1^{\text {st }}$ choice \\
\hline 20 & $6(5-6)$ & $5(5-5.5)$ & $6.25(6-6.5)$ & $1^{\text {st }}$ choice \\
\hline 21 & $6(5.5-6)$ & $5(5-5.5)$ & $6(5-6.5)$ & $2^{\text {nd }}$ choice \\
\hline 22 & $6.5(6-6.5)$ & $5.75(5-6)$ & $7(6.5-7.5)$ & $1^{\text {st }}$ choice \\
\hline 23 & $5(4-5)$ & $4.5(4-4.5)$ & $5.5(5.5-6.5)$ & Extra \\
\hline 24 & $4.5(4-4.5)$ & $4.5(4-4.5)$ & $5.5(5-6)$ & Extra \\
\hline 25 & $5.5(5-5.5)$ & $4.5(4-4.5)$ & $5.5(5-6)$ & Extra \\
\hline 26 & $6(5.5-6)$ & $5.5(5-6)$ & $6(5.5-6.5)$ & Extra \\
\hline 27 & $5.5(5-6)$ & $5(5-6)$ & $6(5-6.5)$ & Extra_wd \\
\hline 28 & $4.5(4-5)$ & $5(5-5.5)$ & $5.5(5-6.5)$ & Extra_wd \\
\hline 29 & $5.5(5-5.5)$ & $4.5(4-4.5)$ & $6(5-6.5)$ & Extra_wd \\
\hline 30 & $4(4-4.5)$ & $5.5(5-5.5)$ & $5(5-5.5)$ & $2^{\text {nd }}$ choice \\
\hline 31 & $6(6-6.5)$ & $4.5(4.5-5)$ & $7(6.5-7)$ & Extra_wd \\
\hline 32 & $6(6-6.5)$ & $4.5(4-4.5)$ & $6(6-6.5)$ & Extra_wd \\
\hline 33 & $2.75(2-3.5)$ & $2.25(2-2.5)$ & $3.5(3-4)$ & Extra_wd \\
\hline 34 & $2.75(2-3.5)$ & $2.25(2-3)$ & $3.5(3-4)$ & Extra_wd \\
\hline 35 & $4.75(4.5-5.5)$ & $5.25(5-6)$ & $4.25(4-4.5)$ & $1^{\text {st }}$ choice \\
\hline 36 & $6.5(6-6.5)$ & $5.5(5-5.5)$ & $7(6-7.5)$ & $1^{\text {st }}$ choice \\
\hline 37 & $4(4-4.5)$ & $4.25(4-4.5)$ & $4(3.5-5)$ & $1^{\text {st }}$ choice \\
\hline 38 & $5.5(5-6)$ & $5.75(5-6)$ & $6(5-6.5)$ & $2^{\text {nd }}$ choice \\
\hline 39 & $4.25(4-5)$ & $5(5-5.5)$ & $5(4.5-6)$ & Extra \\
\hline 40 & $6.5(6-6.5)$ & $5(5-5.5)$ & $6(5-6.5)$ & Extra \\
\hline $41-44^{\mathrm{b}}$ & - & - & - & $\mathrm{NC}$ \\
\hline
\end{tabular}

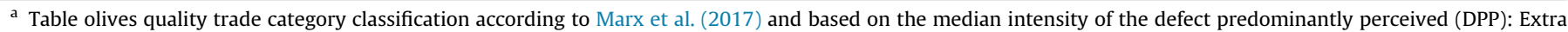

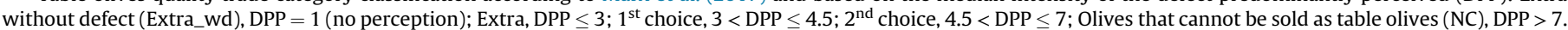

${ }^{\mathrm{b}}$ Inedible samples (i.e., unsuitable for consumption and for that reason the gustatory attributes were not evaluated by the sensory panel).

regression (MLR) models were used to verify the performance of the E-tongue as a taste sensor device for distinguishing aqueous standard solutions that mimicked table olives' gustatory attributes (acid, bitter and salty) and to quantify the median intensities of the basic tastes perceived in table olives by the sensory panel. In both cases, the best subsets of independent predictors (i.e., sensors) were established between the potentiometric sensor signals recorded (40 signals for the standard solutions and 80 signals for the table olives, being 40 signal profiles recorded for aqueous olive pastes and the other 40 for brines) using a meta-heuristic simulated annealing (SA) variable selection algorithm (Bertsimas \& Tsitsiklis, 1993; Cadima, Cerdeira, \& Minhoto, 2004; Kirkpatrick, Gelatt, \& Vecchi, 1983). The E-tongue-LDA-SA and the E-tongue-MLR-SA were evaluated using two cross-validation (CV) variants: leaveone-out (LOO-CV), known to be an over-optimistic procedure; and, repeated $K$-fold (repeated $K$-fold-CV) techniques. For the latter, data was randomly split into $K$ folds, being each of the folds left out in turn and the other $K-1$ folds used to train the model. The held out fold is used for test purposes and the quality of the predictions is assessed using the percentage of correct classifications. The $K$ estimates are averaged to get the overall resampled estimate (Kuhn \&
Johnson, 2013). In this work the number of folds was set equal to 4 , enabling the random formation of internal validation subsets (for each gustatory group) with $25 \%$ of the initial data, allowing bias reduction. The procedure was repeated 10 times for putting the model under stress. The repeated $K$-fold-CV technique allows reducing the uncertainty of the estimates, by evaluating the predictive performance of the models established using $4 \times 10$ random sub-sets for internal validation (i.e., 40 total resamples). The possibility of using the selected E-tongue-MLR-SA models (for both CV variants) as complementary tools for the quantification of acid, bitter and salty sensations perceived in the table olives was further checked, as suggested by Roig and Thomas (2003). The checking technique involved the establishment of the 95\% intervals of confidence (IC) for the slope and intercept values of the single linear regression (LR) obtained by plotting the gustatory intensities predicted by the E-tongue-MLR-SA models versus the respective intensities perceived by the trained sensory panel. The proposed Etongue based approach could be foreseen as a satisfactory tool if the 95\% IC contained the theoretic values of "zero" and "one" for the intercept and slope values, respectively (Roig \& Thomas, 2003).

All statistical analysis were performed using the Subselect 
(Cadima, Cerdeira, Silva, \& Minhoto, 2012; Cadima et al., 2004) and MASS (Venables \& Ripley, 2002) packages of the open source statistical program R (version 2.15.1), at a 5\% significance level.

\section{Results and discussion}

\subsection{E-tongue discrimination of basic gustatory attributes: aqueous standard solution mimicking acid, bitter and salty sensations}

The E-tongue performance as a taste sensor device was evaluated using aqueous standard solutions (Table 2), which mimicked the 3 basic gustatory attributes usually assessed during table olives' sensory analysis as recommended by the IOC regulations (IOC, 2011): acid sensation (citric and tartaric acids), bitter taste (caffeine and anhydrous quinine) and salty sensation (sodium and potassium chloride), prepared with commercial mineral water to mimic the conditions used during the sensory panel training process. For the standard compounds studied, the potentiometric signals recorded with the 20 different lipid-polymeric membranes (S1:1 to S1:20, mixtures of 4 additives $\times 5$ plasticizers) and respective replicas (S2:1 to $S 2: 20$ ) showed a linear (positive or negative) dependency with the decimal logarithm of the concentration $\left(0.923 \leq R^{2} \leq 0.999\right)$. For the substances used to mimic the acid basic taste, both positive and negative sensitivities were observed (citric acid: signals varying from -50 to $+210 \mathrm{mV}$, sensitivities ranging from +9 to $+20 \mathrm{mV} /$ decade and $0.831 \leq R^{2} \leq$ 0.950; tartaric acid: -80 to $+210 \mathrm{mV},-20$ to $-0.01 \mathrm{mV} /$ decade and $0.830 \leq R^{2} \leq 0.971$ ). For the bitter taste, only positive sensitivities were observed for both chemical compounds used (caffeine: -75 to $+197 \mathrm{mV},+20$ to $+31 \mathrm{mV} /$ decade and $0.830 \leq R^{2} \leq 0.831$; quinine: -67 to $+191 \mathrm{mV},+19$ to $+48 \mathrm{mV} /$ decade and $0.830 \leq$ $\left.R^{2} \leq 0.980\right)$. It should be remarked that the sensitivities obtained in this work for quinine are similar to those reported by Veloso et al. (2016), for aqueous solutions prepared with deionized water. Finally, for the salty taste, only negative sensitivities were found for the two substances studied (potassium chloride: -200 to $+150 \mathrm{mV},-51$ to $-14 \mathrm{mV} /$ decade and $0.840 \leq R^{2} \leq 0.980$; sodium chloride: -220 to $+150 \mathrm{mV},-39$ to $-11 \mathrm{mV} /$ decade and 0.814 $\leq R^{2} \leq 0.970$ ). Moreover, it was found that the potentiometric signals recorded by each sensor towards a specific taste compound, had satisfactory repeatability with relative standard deviation percentage (RSD\%) values lower than $12 \%$ (acid taste: $0.8 \% \leq$ RSD $\%$ $\leq 12 \%$; bitter taste: $1.2 \% \leq \mathrm{RSD} \% \leq 12 \%$; and, salty taste: $0.1 \% \leq \mathrm{RSD}$ $\% \leq 11 \%$ ). The different electrochemical behaviors found towards the standard chemical compounds could be attributed to the differences in the composition of the lipid-plasticizer-PVC membranes, which may lead to hydrophobic or hydrophilic interactions depending on the $\mathrm{pH}$, or to the fact that each sensor membrane behaves like a polymer, and so its permeability depends on the solutes' molecular volume (Marx et al., 2017).

Furthermore, the potentiometric signal profiles confirmed the capability of the E-tongue-LDA-SA procedure for discriminating aqueous standard solutions that mimic acid, bitter and salty basic tastes, which are evaluated during table olives' sensory analysis. An E-tongue-LDA-SA model with 2 significant functions ( $P$-value $<$ 0.0001 ; explaining $92.5 \%$ and $7.5 \%$ of the original data variability) based on the signals of 5 sensors (S1:9, S1:14, S2:4, S2:14, e S2:16) was established. The model allowed the correct classification of $100 \%$ of the original grouped data (Fig. 1) and $98 \%$ of the standard solutions for LOO-CV procedure (i.e., only 1 acid solution misclassified as bitter). As can be inferred from Fig. 1, total differentiation of the gustatory attributes evaluated is achieved, although with a slight overplot between salty and bitter tastes. The predictive performance of the E-tongue-LDA-SA model was further tested using the repeated $K$-fold-CV procedure ( 4 folds $\times 10$ repetitions). The best predictive classification results were obtained using a model also based on the same 5 sensors (correct classifications of $98 \% \pm 3 \%$, ranging from $91 \%$ e $100 \%$ for the 40 evaluations performed).

\subsection{Intensities of the descriptive gustatory attributes perceived in table olives by the trained sensory panel}

From the 44 table olive samples studied, 4 of them, which were previously classified as olives that cannot be sold as table olives (samples 41 to 44, DPP > 7, in a 11-scale grade, according to Marx et al. (2017)), were not edible for consumption and so, were not tasted by the panelists. So, in this study, the descriptive gustatory attributes (i.e., acid, bitter and salty) could only be evaluated for 40 table olive samples (samples 1 to 40 , described by Marx et al.

\section{Table 4}

Predictive capability of the E-tongue-MLR-SA models established to quantify the median intensities of 3 descriptive gustatory attributes (acid, bitter and salty) evaluated by a trained panelists, as recommended by the IOC (2011), during the sensory analysis of commercial table olives.

\begin{tabular}{|c|c|c|c|}
\hline \multirow{3}{*}{$\begin{array}{l}\text { Descriptive gustatory attribute } \\
\text { (IOC, 2011) }\end{array}$} & \multicolumn{3}{|c|}{ E-tongue-MLR-SA models ${ }^{\mathrm{a}}$} \\
\hline & \multirow[t]{2}{*}{$\mathrm{N}^{\circ}$ of signals ${ }^{\mathrm{b}}$} & \multicolumn{2}{|c|}{ Determination coefficient $\left(R^{2}\right)$} \\
\hline & & ${\mathrm{LOO}-\mathrm{CV}^{\mathrm{C}}}$ & Repeated $K$-fold-CV \\
\hline $\begin{array}{l}\text { Acid basic taste } \\
\text { Bitter basic taste } \\
\text { Salty basic taste }\end{array}$ & $\begin{array}{l}21^{\mathrm{e}} \\
25^{\mathrm{f}} \\
22^{\mathrm{g}}\end{array}$ & $\begin{array}{l}0.987 \\
0.997 \\
0.988\end{array}$ & $\begin{array}{l}0.97 \pm 003 \\
0.98 \pm 004 \\
0.96 \pm 004\end{array}$ \\
\hline \multicolumn{4}{|c|}{$\begin{array}{l}\text { a Multivariate linear regression (MLR) model based on the sub-sets of potentiometric signals, established using the simulated annealing (SA) algorithm, selected } \\
\text { among the } 80 \text { possible signal profiles obtained with the electronic tongue (E-tongue) during the analysis of olive pastes (40 sensor signals) and brines ( } 40 \text { sensor } \\
\text { signals). } \\
\text { b Number of signals included in the E-tongue-MLR-SA model, selected from the } 80 \text { electrochemical signals recorded by E-tongue during analysis of each olive } \\
\text { paste ( } 40 \text { signals referring to } 40 \text { sensors LE) and respective brine ( } 40 \text { signals related to } 40 \text { sensors the E-tongue). } \\
\text { c LOO-CV: leave-one-out cross validation procedure. } \\
\text { d Repeated } K \text {-fold-CV: cross-validation procedure with } 4 \text { folds, ensuring that at least } 25 \% \text { of the original data are used for internal validation, and } 10 \text { repetitions. } \\
\text { e E-tongue signals included in the E-tongue-MLR-SA model: } 7 \text { recorded signals during the analysis of the brines (S1:3, S1:7, S1:9, S2:1, S2:14, S2:16 and S2:17) and } \\
\text { the other related to the response of } 14 \text { sensors during the analysis of the aqueous olive paste (S1:6, S1:8, S1:14, S1:17, S1:18, S2:3, S2:4, S2:11 to S2:13, S2:15, S2:17, } \\
\text { S2:19 and S2:20). } \\
\text { f E-tongue signals included in the E-tongue-MLR-SA model: } 13 \text { recorded signals during the analysis of the brines (S1:2, S1:9, S1:18, S2:1, S2:4, S2:5, S2:10, S2:12 } \\
\text { to S2:15, S2:17 and S2:19) and the other related to the response of } 12 \text { sensors during the analysis of the aqueous olive paste (S1:2, S1:4, S1:6, S1:7, S1:9, S1:14, S1:17, } \\
\text { S1:18, S2:3, S2:9, S2:13 and S2:16). } \\
\text { g E-tongue signals included in the E-tongue-MLR-SA model: } 14 \text { recorded signals during the analysis of the brines (S1:5, S1:6, S1:9, S1:11, S1:12, S1:15, S2:1, S2:2, } \\
\text { S2:5, S2:8, S2:10, S2:13, S2:14 and S2:16) and the other related to the response of } 8 \text { sensors during the analysis of the aqueous olive paste (S1:4, S1:5, S1:6, S1:8, } \\
\text { S1:9, S1:16, S2:1 and S2:14). }\end{array}$} \\
\hline
\end{tabular}



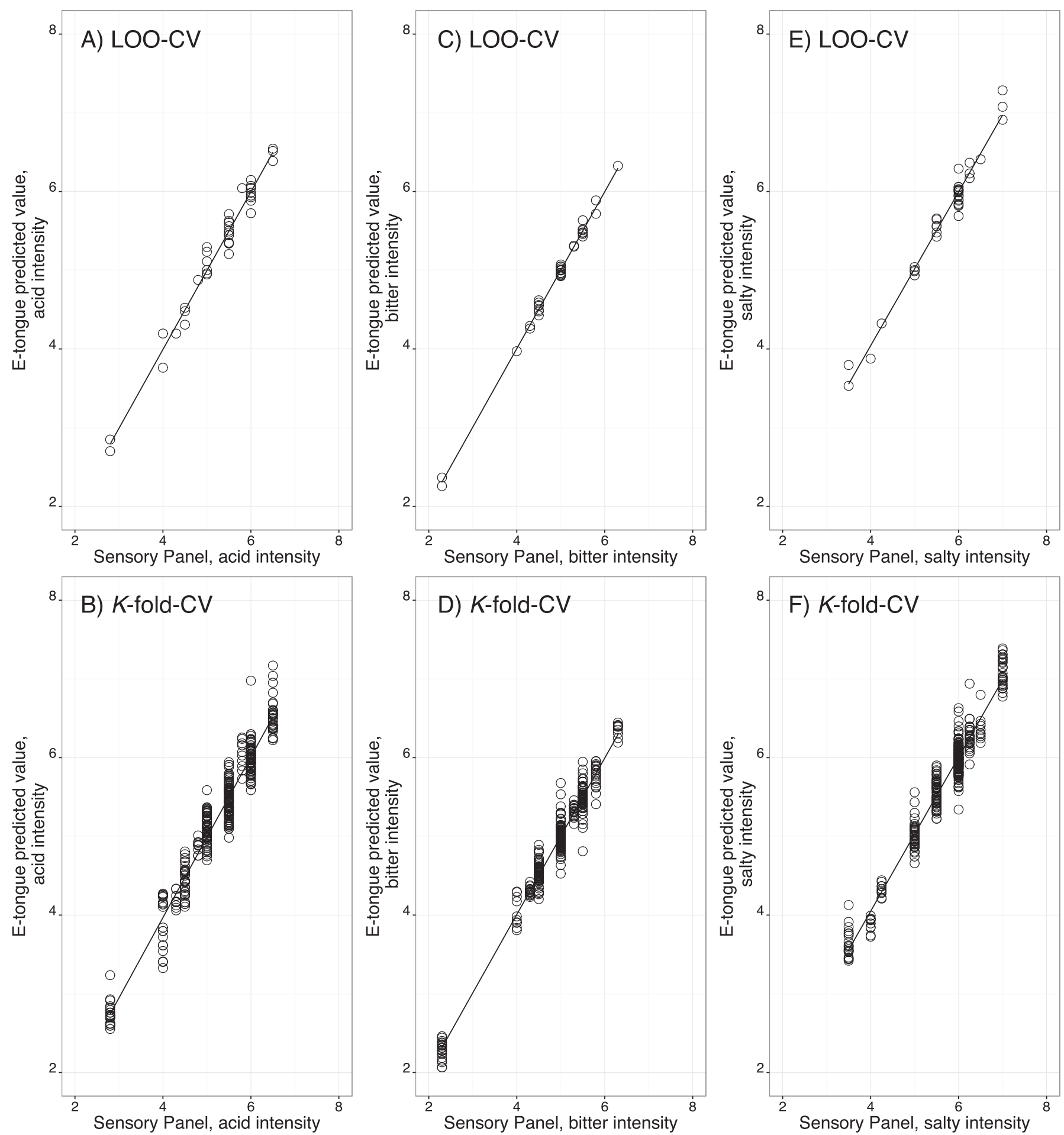

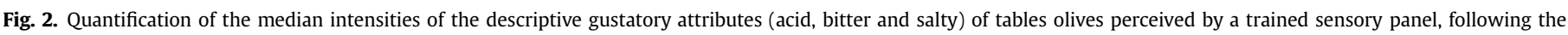

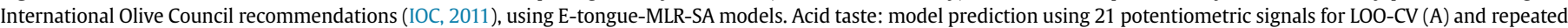

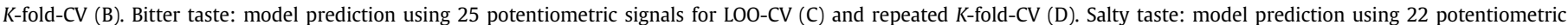
signals for LOO-CV (E) and repeated $K$-fold-CV (F).

(2017)). Each sample (from those 40) and the brine were evaluated by the trained sensory panel according to the IOC recommendations (IOC, 2011). The median intensities of the descriptive gustatory attributes perceived in the table olive samples by the panelists are shown in Table 3, together with interval of the intensities perceived. It is also reported the trade quality sensory classification of each sample according to Marx et al. (2017). The narrow variation found in the intensities perceived by the 8 panelists regarding each basic taste detected in a sample (maximum observed variation equal or lower than 1.5 intensity units) showed the capability of the sensory panel for evaluating acid, bitter and salty sensations. According to the sensory analysis, the 3 basic tastes were perceived in 
Table 5

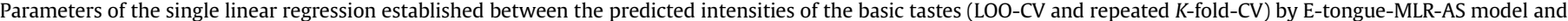

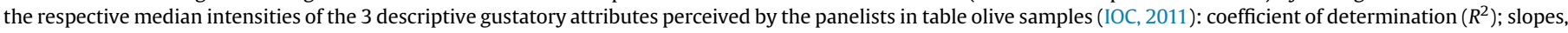
intercept values and respective confidence intervals (CI) at 95\%.

\begin{tabular}{|c|c|c|c|c|c|c|}
\hline \multirow[t]{3}{*}{ Single regression line parameters } & \multicolumn{6}{|c|}{ Descriptive gustatory attributes evaluated during table olives sensory analysis (IOC, 2011) } \\
\hline & \multicolumn{2}{|l|}{ Acid taste } & \multicolumn{2}{|l|}{ Bitter taste } & \multicolumn{2}{|l|}{ Salty taste } \\
\hline & LOO-CV & Repeated $K$-fold-CV ${ }^{\mathrm{d}}$ & LOO-CV & Repeated $K$-fold-CV & LOO-CV & Repeated $K$-fold-CV ${ }^{\mathrm{d}}$ \\
\hline$R^{2}$ & 0.987 & 0.967 & 0.997 & 0.978 & 0.988 & 0.955 \\
\hline Slope & 1.002 & 1.017 & 0.997 & 0.996 & 0.977 & 0.987 \\
\hline Slope $\mathrm{CI}^{\mathrm{a}}$ & {$[0.949,1.055]$} & {$[0.998,1.035]$} & {$[0.974,1.020]$} & {$[0.981,1.011]$} & {$[0.927,1.028]$} & {$[0.966,1.008]$} \\
\hline Intercept & -0.014 & -0.098 & 0.016 & 0.016 & 0.133 & 0.078 \\
\hline Intercept $\mathrm{CI}^{\mathrm{b}}$ & {$[-0.300,0.271]$} & {$[-0.197,0.001]$} & {$[-0.098,0.131]$} & {$[-0.057,0.089]$} & {$[-0.158,0.423]$} & {$[-0.044,0.199]$} \\
\hline
\end{tabular}

a $95 \%$ slope confidence interval.

b $95 \%$ intercept confidence interval.

c LOO-CV (leave-one-out cross-validation).

d Repeated $K$-fold-CV ( 4 folds $\times 10$ repeats).

all the 40 edible table olive samples, ranging from low to moderate intensities (intensities from 2.75 to 7 for a 11-units scale). Moreover, the one-way ANOVA pointed out that table olives of the 5 quality trade categories had similar acid and salty median perceived intensities ( $P$-values $=0.2511$ and 0.5708 , respectively). Regarding the bitter taste, the median perceived intensities significantly differ with the quality trade category ( $P$ value $=0.0059$, one-way ANOVA) having tables olives classified as extra without defects the lowest bitterness, mainly when compared to those classified as $1^{\text {st }}$ choice and $2^{\text {nd }}$ choice $(P$-values $=0.0060$ and 0.0106, respectively; multiple comparison post-hoc Tukey's test). Furthermore, although not statistically significant, for the samples evaluated in this study, the perceived bitter sensation increased with the decrease of the table olives' sensory quality.

\subsection{E-tongue quantification of the median intensities of gustatory attributes perceived in table olives}

The potentiometric fingerprint of table olives, more concretely, aqueous olive pastes and respective brines solutions, recorded by an E-tongue were analyzed using chemometric methods, aiming to quantify the sensory intensities of the basic tastes (acid, bitter and salty) perceived by trained panelists during the sensory analysis of commercial table olives (Table 3). The matrix complexity (olive plus brine) together with the different technological procedures applied, the different olive cultivars and aromatic compounds used, makes this task a challenge from an instrumental and modeling point of view. Moreover, since the majority of the table olives (33 of the 40 samples) had 1 to 3 of a total of 5 sensory defects (butyric, putrid, zapateria, musty and/or wine-vinegary negative sensations) (Marx et al., 2017), the perception and quantification of the intensities of the descriptive gustatory attributes by the E-tongue could be viewed as a hard task. Based on the signal profiles gathered during the analysis of the aqueous olive pastes and respective brines, MLR models were established based on the best sub-sets of signals (21-25 sensor signals), selected by the SA algorithm, and their performances evaluated using two cross-validation variants (LOO-CV and repeated $K$-fold-CV with 4 folds $\times 10$ repetitions). The results of the predictive performances of the E-tongue-MLR-SA models regarding the quantification of the median intensities of the perceived gustatory sensations are given in Table 4, including the number and type of sensors used in the models for assessing each gustatory sensation. The overall determination coefficients obtained for LOO-CV $\left(0.987 \leq R^{2} \leq 0.997\right)$ and repeated $K$-fold-CV $\left(0.96 \pm 0.04 \leq R^{2} \leq 0.98 \pm 0.04\right)$ procedures pointed out the satisfactory capability of the proposed electrochemical approach for quantifying the median intensities of the acid, bitter and salty sensations perceived in table olives. It should be also remarked that some sensors are included in all the E-tongue-MLR-SA models, which was expected due to their low specificity towards specific tastes, although some other sensors (in less number) seemed more selective towards a specific taste, since they were only included in one model. Last, the quality of the regression results for LOO-CV and repeated $K$-fold-CV can also be verified from Fig. 2 . As expected, for repeated $\mathrm{K}$-fold-CV, the predicted median intensities of the 3 descriptive gustatory attributes, obtained with the E-tongueMLR-SA models, show a higher variability compared to the more optimistic LOO-CV technique. It should also be emphasized that, for the $K$-fold-CV procedure, the variability found for each taste sensation (symbols " $\bigcirc$ " in Fig. 2), is acceptable although higher compared to the interval range of the individual intensity evaluation performed by the trained panelists during the sensory analysis of each table olive sample (Table 3). Even so, it is possible to conclude that the E-tongue-MLR-SA models can effectively mimic the overall performance of the trained sensory panel.

Finally, as suggested by Roig and Thomas (2003), the applicability of the E-tongue-MLR-SA models as a complementary helpful tool for assessing table olives' gustatory attributes, was evaluated. For that, a single linear regression was established between the intensities predicted by the E-tongue-MLR-SA and the median intensities perceived by the sensory panel, and it was verified if the slope and intercept values were statistically equal to one and zero (as theoretically expected for a perfect linear fit), respectively. Table 5 shows the parameters of the single linear regressions established as well as the determination coefficients $\left(R^{2}\right)$, the slope and intercept values as well as the respective 95\% confidence intervals, for both $\mathrm{LOO}-\mathrm{CV}$ and repeated $\mathrm{K}$-fold-CV techniques, for each of the 3 table olives' gustatory attributes. Since the confidence intervals for the slope and intercept contained the values theoretical expected values (one and zero, respectively), it was demonstrated that, at $5 \%$ significance level, the proposed taste sensor could perform equally well as a trained sensory panel, confirming the expected potential of the proposed E-tongue-MLR-SA technique as a sensory practical tool for assessing the intensities of acid, bitter and salty tastes perceived in table olives. This achievement has even more impact, considering the complex mixture of flavors and tastes of table olives arising from the addition of several spices during their production and from the development of several sensory negative sensations, commonly found in table olives in different intensity levels.

\section{Conclusions}

An analytical methodology using an electronic tongue in combination with qualitative and quantitative multivariate linear analysis was proposed as a taste sensor approach to discriminate 
and quantify descriptive gustatory attributes (acid, bitter and salty) that are assessed during table olives sensory analysis, according to the directives of the International Olive Council. The promising results obtained and described in this work, clearly point out that the potentiometric signal profiles gathered from cross-sensitivity lipid polymeric membranes may be used as an accurate gustatory fingerprint of the acid, bitter and salty basic tastes of table olives. Indeed, this study shows that an instrumental multisensor device fused with appropriate chemometric tools, may be a complementary technique to the complex, costly and time-consuming official table olive sensory assessment, which must be performed by official sensory panels. Despite the satisfactory overall performance achieved with the electronic tongue, a fusion of different sensorbased devices is foreseen, aiming to fully evaluate the broad sensory sensations perceived by a trained sensory panel.

\section{Acknowledgments}

This work was financially supported by Project POCI-01-0145FEDER-006984 - Associate Laboratory LSRE-LCM and by Project UID/QUI/00616/2013 - CQ-VR both funded by FEDER - Fundo Europeu de Desenvolvimento Regional through COMPETE2020 Programa Operacional Competitividade e Internacionalização (POCI) - and by national funds through FCT - Fundação para a Ciência e a Tecnologia, Portugal. Strategic funding of UID/BIO/ 04469/2013 unit is also acknowledged. Nuno Rodrigues thanks FCT, POPH-QREN and FSE for the Ph.D. Grant (SFRH/BD/104038/2014).

\section{References}

Apetrei, C., Apetrei, I. M., Villanueva, S., de Saja, J. A., Gutierrez-Rosales, F., et al. (2010). Combination of an e-nose, an e-tongue and an e-eye for the characterisation of olive oils with different degree of bitterness. Analytica Chimica Acta, 663, 91-97.

Apetrei, C., Ghasemi-Vernamkhasti, M., \& Apetrei, I. M. (2016). Chapter 27 - olive oil and combined electronic nose and tongue. In M. L. Rodríguez-Méndez (Ed.), Electronic noses and tongues in food science (pp. 277-289). London: Academic Press.

Bertsimas, D., \& Tsitsiklis, J. (1993). Simulated annealing. Statistical Science, 8, 10-15.

Blana, V. A., Polymeneas, N., Tassou, C. C., \& Panagou, E. Z. (2016). Survival of potential probiotic lactic acid bacteria on fermented green table olives during packaging in polyethylene pouches at 4 and $20^{\circ} \mathrm{C}$. Food Microbiology, 53, 71-75.

Borràs, E., Ferré, J., Boqué, R., Mestres, M., Aceña, L., Calvo, A., et al. (2016). Olive oil sensory defects classification with data fusion of instrumental techniques and multivariate analysis (PLS-DA). Food Chemistry, 203, 314-322.

Cadima, J., Cerdeira, J. O., \& Minhoto, M. (2004). Computational aspects of algorithms for variable selection in the context of principal components. Computational Statistics \& Data Analysis, 47, 225-236.

Cadima, J., Cerdeira, J. O., Silva, P. D., \& Minhoto, M. (2012). The subselect R package. http://cran.rproject.org/web/packages/subselect/vignettes/subselect.pdf Accessed 15/02/2016.

Campus, M., Sedda, P., Cauli, E., Piras, F., Comunian, R., Paba, A., et al. (2015). Evaluation of a single strain starter culture, a selected inoculums enrichment, an natural microflora in the processing of Tonda di Cagliari natural table olives: Impact on chemical, microbiological, sensory and texture quality. LWT - Food Science and Technology, 64, 671-677.

Cano-Lamadrid, M., Hernández, F., Corell, M., Burló, F., Legua, P., Moriana, A., et al. (2016). Antioxidant capacity, fatty acids profile, and descriptive sensory analysis of table olives as affected by deficit irrigation. Journal of the Science of Food and Agriculture. http://dx.doi.org/10.1002/jsfa.7744.

De Angelis, M., Campanella, D., Cosmai, L., Summo, C., Rizzello, C. G., \& Caponio, F.
(2015). Microbiota and metabolome of un-started and started Greek-type fermentation of Bella di Cerignola table olives. Food Microbiology, 52, 18-30.

Dias, L. A., Peres, A. M., Veloso, A. C. A., Reis, F. S., Vilas-Boas, M., Machado, et al. (2009). An electronic tongue taste evaluation: Identification of goat milk adulteration with bovine milk. Sensors and Actuators B. - Chemical, 136, 209-217.

Dias, L. G., Rodrigues, N., Veloso, A. C. A., Pereira, J. A., \& Peres, A. M. (2016). Monovarietal extra virgin olive oils classification: A fusion of human sensory attributes and an electronic tongue. European Food Research and Technology, 242, 259-270.

Dias, L. G., Sequeira, C., Veloso, A. C. A., Sousa, M. E. B. C., \& Peres, A. M. (2014). Evaluation of healthy and sensory indexes of sweetened beverages using an electronic tongue. Analytica Chimica Acta, 848, 32-42.

Iiyama, S., Yahiro, M., \& Toko, K. (2000). Measurements of soy sauce using taste sensor. Sensors and Actuators B: Chemical, 66, 205-206.

IOC, International Olive Council. (2011). Method for the sensory analysis of table olives. http://www.internationaloliveoil.org/estaticos/view/224-testing-methods, 2011 (Accessed 10-02-16).

IOC, International Olive Oil Council. (2004). 'Trade standards applying to table olives.' IOC/OT/NC no. 1. Madrid, Spain: International Olive Oil Council.

Kirkpatrick, S., Gelatt, C. D., \& Vecchi, M. P. (1983). Optimization by simulated annealing. Science, 220, 671-680.

Kobayashi, Y., Habara, M., Ikezazki, H., Chen, R., Naito, Y., \& Toko, K. (2010). Advanced taste sensors based on artificial lipids with global selectivity to basic taste qualities and high correlation to sensory scores. Sensors, 10, 3411-3443.

Kuhn, M., \& Johnson, K. (2013). Applied predictive modeling. New York: Springer Science Business Media.

Lanza, B., \& Amoruso, F. (2016). Sensory analisys of natural table olives: Relationship between appearance of defect and gustatory-kinaesthetic sensation changes. LWT - Food Science and Technology, 68, 365-372.

López-López, A., Bautista-Gallego, J., Moreno-Baquero, J. M., \& GarridoFernández, A. (2016). Fermentation in nutrient salt mixtures affects green Spanish-style Manzanilla table olive characteristics. Food Chemistry, 211, $415-422$.

Martorana, A., Alfonzo, A., Settanni, L., Corona, O., La Croce, F., Caruso, T., et al. (2016). Effect of the mechanical harvest of drupes on the quality characteristics of green fermented table olives. Journal of the Science of Food and Agriculture, 96, 2004-2017.

Marx, Í., Rodrigues, N., Dias, L. G., Veloso, A. C. A., Pereira, J. A., Drunkler, D. A., et al. (2017). Sensory classification of table olives using an electronic tongue: Analysis of aqueous pastes and brines. Talanta, 162, 98-106.

Meilgaard, M., Civille, G. V., \& Carr, B. T. (2007). Sensory evaluation techniques (4th ed.). Boca Raton: CRC Press.

Mizota, Y., Matsui, H., Ikeda, M., Ichihashi, N., Iwatsuki, K., \& Toko, K. (2009). Flavor evaluation using taste sensor for UHT processed milk stored in cartons having different light permeabilities. Milchwissenschaft, 64, 143-146.

Panagou, E. Z., Sahgal, N., Magan, N., \& Nychas, G.-J. E. (2008). Table olives volatile fingerprints: Potential of an electronic nose for quality discrimination. Sensors and Actuators B: Chemical, 134, 902-907.

Randazzo, C. L., Todaro, A., Pino, A., Pitino, I., Corona, O., Mazzaglia, A., et al. (2014). Giarraffa and Grossa di Spagna naturally fermented table olives: Effect of starter and probiotic cultures on chemical, microbiological and sensory traits. Food Research International, 62, 1154-1164.

Rodríguez-Gómez, F., López-López, A., Romero-Gil, V., Arroyo-López, F. N., MorenoBaquero, J. M., Garrido-Fernández, A., et al. (2014). Effect of post-fermentation storage on Spanish-style green Manzanilla olives. LWT - Food Science and Technology, 57, 789-793.

Rodríguez-Méndez, M. L., Apetrei, C., \& de Saja, J. A. (2010). Chapter 57-Electronic tongues purposely designed for the organoleptic characterization of olive oils. In V. R. Preedy, \& R. R. Watson (Eds.), Olives and olive oil in health and disease prevention (pp. 525-532). London: Academic Press.

Roig, B., \& Thomas, O. (2003). UV monitoring of sugars during wine making. Carbohydrate Research, 338, 79-83.

Toko, K. (2013). Biochemical Sensors: Mimicking gustatory and olfactory senses. Boca Raton: CRC Press.

Veloso, A. C. A., Dias, L. G., Rodrigues, N., Pereira, J. A., \& Peres, A. M. (2016). Sensory intensity assessment of olive oils using an electronic tongue. Talanta, 146, 585-593.

Venables, W. N., \& Ripley, B. D. (2002). Modern applied statistics with S (statistics and computing) (4th ed.). , New York: Springer. 